\title{
CONSPECTVS POETARVM
}

L. ACCIVS

Praetertatae

Tragica

Varia

L. AFRANIVS

Togatae

ANONYMI VERSVS

APRISSIVS

Atellana

AQVILIVS

Comica

ATILIVS

Comicum

STATIVS CAECILIVS

Comicà

CARMINA VETVSTISSIMA 3

COMICI INCERTI

Q. ENNIVS

Annales

Comica

Epicharmus

Hedyphagetica

Praetextatae

Saturae

Scenica incerta

Tragica

Varia

EPIGRAMMA

Naevii

Pacurii

Plauti

A. FVRTVS ANTIAS

Poemats

HOSTIVS

Belhum Histricum

TVVENTIVS

Comica

D. LABERTVS

Mimi

LICNIVS IMBREX

Comicom

\section{LIVIVS ANDRONICVS}

Comica

Odyssia

Tragica

C. LVCILIVS

Saturae

Q. LVTATIVS CATVLVS

Epigrammata

CN. NAEVIVS

Bellum Poenicum

Comica

Praetextatae

Tragica

Novivs

Atellanae

M. PACVVIVS

Praetextatae

Tragica

POMPILIVS

17 Epigrammata 149

46 Tragicum 149

49 L. POMPONIVS

49 Atellanae

PORCIVS LICINVS

PVBLILIVS SYRVS

Mimi

Sententiae

T. QVINCTIVS ATTA

Togatae

SATVRNII

TITINIVS

Togatae

TRABEA

Comica

TRAGICI INCERTI

SEX. TVRPILIVS

Comica

66 VALEKIVS AEDITVVS

Epigrammata

156

O. VALERIVS SORANVS 146 VOLCACIVS SEDIGITVS

De poetis

\section{9}

7

102

149

9

14

12

154

58

49

149
149

150

147

160

160

95

3

92

65

89

67

148

$14^{8}$ 